\title{
MORTALIDADE POR AFOGAMENTO NO MUNICÍPIO DE SALVADOR, 1980 A 1994*
}

\author{
Iacira Magalhães Oliveira ${ }^{1}$, Lígia Maria Vieira da Silva ${ }^{1}$, Maria da Conceição \\ Nascimento Costa ${ }^{1}$, Jairnilson Silva Paim ${ }^{1}$
}

\begin{abstract}
Resumo
Foi realizado um estudo com o objetivo de descrever a evolução da mortalidade por afogamento em Salvador no periodo de 1980-1994, suas características demográficas e a distribuição espacial nos anos de 1991 e 1994. Foram utilizados dados secundários das Estatisticas de Mortalidade do Ministério da Saúde para descrição da evolução e fotocópias das Declaraçôes de Óbito do Centro de Informaçôes em Banco de Dados da Secretaria Estadual de Sauide da Bahia. As informaçoes foram complementadas a partir dos registros do Instituto Médico Legal Nina Rodrigues. No periodo estudado houve um decréscimo da taxa de mortalidade por afogamento de 7,9 para 5,3 por 100.000 habitantes, bem como da proporção desses óbitos em relação às causas externas. Houve uma predominància da mortalidade entre a população masculina, estudantes e pessoas com o primeiro grau de escolaridade. Os óbitos por afogamento foram mais freqüentes no litoral de Salvador durante os meses de verão. Há uma desigualdade no risco de morrer entre residentes dos diversos Distritos Sanitários da cidade. Os autores discutem possiveis estratégias para o controle e prevenção do problema.
\end{abstract}

Palavras Chaves: Afogamento; Mortalidade; Causas Externas.

\section{Summary}

The aim of this study was to describe the evolution of deaths caused by drowning in Salvador in the period between 1980-1994, some demographic characteristics of those deaths and it's spacial distribution in Salvador, a city situated in the northeast of Brazil. The data was obtained from Death Certificates, official publications and from the Medical Legal Institute's archives. In the period studied there was a reduction of the mortality rate by drowning of 7.9 to 5.3 per 100.000 inhabitants, as well as in the proportion in relation to external causes of death. The mortality rates were higher among males, students and people with elementary education, during the summer months. There were inequalities in the spatial distribution of those deaths in the urban setting of Salvador. The authors suggest specific control preventive measures and public policies directed to the areas with higher death risk.

Key Words: Drowning; Mortality; External Causes.

\footnotetext{
* Trabalbo realizado com apoio OPS/CNPq. Processo $N^{\circ} 100848 / 95-8$ e bolsa de iniciação cientifica.

${ }^{1}$ Instituto de Saude Coletiva/Universidade Federal da Bahia. Endereço para correspondência: Jairnilson Silva Paim Rua Padre Feijó, 29, $4^{\circ}$ andar - Canela, Salvador/Babia/Brasil - Cep: 40.110-170

Tel: (071) 2450544 Fax: (071) 2375856

E-mail: jairnil@ufba.br
} 


\section{Introdução}

Os óbitos vinculados a acidentes $e$ violência, denominados de "causas externas" na Classificação Internacional das Doenças (CID), ${ }^{1}$ representam importante causa de morte em crianças e adolescentes no Brasil. Os óbitos por afogamento e por submersão corresponderam, em 1985, a 16,6\%, 19,2\% e 18,6\% do total de óbitos por "causas externas » para o Brasil, Bahia e Salvador, respectivamente, na faixa etária de 0 a 19 anos. ${ }^{2}$ Em 1990, em Salvador, os afogamentos continuaram situados entre as três principais causas de morte do referido grupo, superadas apenas pelos óbitos por homicidios $e$ acidentes de veiculo. ${ }^{3}$

Estatísticas oficiais da Austrália ${ }^{4}$, do Arizona $^{5}$ e do Alaska ${ }^{6}$ revelam uma queda da mortalidade por esta causa, ao longo dos anos, evidenciando a importância das medidas preventivas e da melhoria na qualidade do atendimento à vitima.

Na América do Sul, o Brasil é o país com a maior faixa litoránea em que o clima quente permite uma grande freqüência de banhistas durante todo o ano. O Rio de Janeiro, sendo região de maior freqüência de banbistas e litoral com características próprias de perigo é área onde ocorrem mais afogamentos em todo litoral brasileiro segundo estudo que caracterizou as vitimas e classificou os diferentes graus de afogamento na tentativa de otimizar o atendimento e diminuir a mortalidade por esta causa. ${ }^{7}$

Os casos de óbito por afogamento têm sido estudados principalmente no que diz respeito aos seus aspectos clínicos e fisiopatológicos. ${ }^{7,8,9}$ Muitos estudos enfocam a importância do afogamento na área pediátrica discutindo medidas preventivas e formas de tratamento para os acidentes ocorridos. A faixa etária de maior ocorrência é a de menores de quatro anos, especialmente aquelas crianças que estão iniciando a marcha, ocorrendo principalmente no sexo masculino e em piscinas. Constitui causa freqüente de morbidade $e$ mortalidade tanto pulmonar como neurológica. ${ }^{8,9,10,11}$ A análise da evolução da mortalidade especifica por esse agravo revelou sua redução no periodo compreendido entre 1976-1980 no Estado do Rio de Janeiro. ${ }^{12}$

Particularmente em Salvador, cidade circundada por praias, não se verificou na literatura pesquisada nenhum trabalho que tratasse especificamente desse tema. Apenas foram identificados estudos realizados sobre mortalidade por causas externas, onde se menciona o problema. ${ }^{2,3}$

O presente estudo pretende descrever a evolução da mortalidade por afogamento no município de Salvador no período de 19801994, e a distribuição espacial dos óbitos e de certas variáveis epidemiológicas nos anos de 1991 e de 1994.

\section{Metodologia}

Foi realizado um estudo descritivo de mortalidade por afogamento no municipio de Salvador, capital do Estado da Babia, situada na Região Nordeste do Brasil. Foi utilizada a divisão do município em 12 Distritos Sanitários feita pela Secretaria de Saúde do Estado da Babia (SESAB), através do agrupamento das 75 Zonas de Informação (ZI). A divisão da cidade em 75 ZI foi desenvolvida pela Companbia de Desenvolvimento da Região Metropolitana de Salvador (CONDER), de acordo com os critérios fisico-urbanisticos, administrativos, de planejamento e de compatibilização com os setores censitários da Fundação Instituto Brasileiro de Geografia e Estatística (FIBGE). A opção pela utilização do Distrito Sanitário como unidade de análise, ao invés da Zona de Informação, decorreu do fato de o número de óbitos por afogamento ser pequeno, ficando rarefeita sua distribuição pelas 75 ZI. Para a descrição da evolução da mortalidade por afogamento em Salvador, entre 1980 e 1994, o número de óbitos foi retirado da publicação "Estatísticas de Mortalidade" do Ministério da Saude. ${ }^{13}$ As estimativas populacionais para $o$ periodo foram obtidas do FIBGE e Ministério da Saúde/Centro de Informaçôes de Saúde (CIS) da SESAB e do Censo Demográfico do IBGE para os anos de 1980 e 1991. 
Para a análise e comparação da distribuição espacial dos óbitos e de certas variáveis epidemiológicas nos anos de 1991 e 1994, foram utilizados os bancos de dados disponiveis no Instituto de Saúde Coletiva da Universidade Federal da Babia (ISC/UFBa)..$^{14}$ A metodologia detalhada dessa investigação foi descrita em estudos anteriores. ${ }^{14,15,16}$

As seguintes variáveis contidas nas Declarações de Óbitos (DO) foram estudadas: sexo, idade, local de residência (segundo DS), mês da ocorrência do óbito, grau de instrução, assim como a ocupação das vitimas. Foram calculadas a mortalidade proporcional (em relação ao total de óbitos por causas externas) $e$ as taxas de mortalidade especificas para afogamento segundo sexo, faixa etária e local de residência da vitima.

Tendo em vista que a maioria das causas de morte violenta se classificava como «não especificada», foi realizada uma segunda fase de coleta de dados nos arquivos do Instituto Médico Legal Nina Rodrigues (IMLNR). Para isso, foi elaborado um questionário a partir das informações contidas no Laudo do Departamento de Polícia Técnica (DPT) do IMLNR e no Laudo do Guia Policial, que traziam maiores detalhes, ou seja, observações que colaboravam para a caracterização do tipo de violência (descrição do exame necroscópico), local em que havia ocorrido o óbito $e$ caracteristicas da violência. A seguir, esses novos dados foram digitados em um banco de dados complementar. A partir dessas informações, procedeu-se à recodificação das DO consideradas preliminarmente como óbitos de causa não especificada. As causas de óbito foram então classificadas pela Classificação Suplementar de Causas Externas (código "E" da Nona Revisão da Classificação Internacional de Doenças' ${ }^{1}$ OMS, 1975). Os óbitos por afogamento foram classificados em E-910.0 a E-910.9.

Os casos de dúvida foram submetidos à avaliação de um especialista em codificação de causa básica da Secretaria de Saúde do Estado da Babia e, quando necessário, foi consultado o Centro Brasileiro de Classificação de Doenças, com sede em São Paulo.

A análise da distribuição espacial dos indicadores foi feita a partir do agrupamento da DS em quartis: mortalidade - baixa, intermediária, elevada e muito elevada. Para a elaboração dos mapas foi utilizado o Programa EPI - MAP versão 2.0.

\section{Resultados}

No periodo de 1980 a 1994 bouve uma diminuição tanto da taxa de mortalidade por afogamento de 7,9 para 5,3 por 100.000 habitantes, quanto da proporção desses óbitos no contexto das causas externas: $12,3 \% \mathrm{em}$ 1980 para 6,0\% em 1994 (Tabela 1). Essa tendência é observada em quase todas as faixas etárias. O grupo de idade de 15 a 19 anos apresenta os maiores coeficientes de mortalidade (com exceção de 1988 que foi o de dez a 14 anos) e o de dez a 19 anos, os maiores percentuais entre os óbitos por causas externas. Há um decréscimo da contribuiçãa dos afogamentos a partir dos 20 anos, nesse grupo de causas.

Após a revisão das DO levadas ao IMLNR, verificou-se que, em 1991, ocorreram em Salvador 102 mortes por afogamento e, em 1994, 96 óbitos. Esses dados diferem dos dados oficiais: 108 óbitos em 1991 e $118 \mathrm{em} 1994$. Nas DO estudadas não havia informações sobre algumas variáveis: em 1991 - mês de ocorrência do óbito (3 casos), local de ocorrência (10 casos), ocupação das vitimas (14 casos); em 1994 - mês de ocorrência (3 casos), faixa etária (4 casos), local de ocorrência (4 casos), gran de instrução (12 casos), local de residência (2 casos) e ocupação da vitima (12 casos).

Mesmo após a revisão das $102 \mathrm{DO}$ do ano de 1991, 83,3\% foram classificadas como não especificadas e em 1994, 100\% foram classificadas como outros - afogamento em: não especificado/piscina/tanque de resfriamento.

$\mathrm{Na}$ Tabela 2 verifica-se uma sobremortalidade masculina da ordem de 8,4 vezes em ambos os anos. Isso se torna mais acentuado na faixa de 15 a 19 anos quando alcança valores de 24,3 em 1991 e 8,3 vezes 
Iacira Magalhães Oliveira e cols

Tabela 1 - Coeficiente de mortalidade e proporção de óbitos por afogamento em relação a causas externas, por faixa etária, Salvador, 1980-1994

\begin{tabular}{|c|c|c|c|c|c|c|c|c|c|c|c|c|c|c|c|c|c|}
\hline \multirow{3}{*}{ Anos } & \multicolumn{17}{|c|}{ Faixa Etária } \\
\hline & \multicolumn{2}{|c|}{$0-4$} & \multicolumn{2}{|c|}{$5-9$} & $10-14$ & \multirow{2}{*}{$\begin{array}{l}15- \\
\mathrm{CM}\end{array}$} & \multirow{2}{*}{$\begin{array}{r}19 \\
\%\end{array}$} & \multicolumn{2}{|c|}{$20-29$} & \multicolumn{2}{|c|}{$30-39$} & \multicolumn{2}{|c|}{$40-49$} & \multicolumn{2}{|c|}{50 e +} & \multicolumn{2}{|c|}{ Total } \\
\hline & $\mathrm{CM}^{(1)}$ & 1) $\%$ & CM & $\%$ & CM \% & & & CM & $\%$ & CM & $\%$ & CM & $\%$ & CM & $\%$ & CM & $\%$ \\
\hline 1980 & 6,9 & 17,9 & 3,5 & 11,5 & $9,0 \quad 26,8$ & 15,6 & 25,0 & 9,8 & 12,7 & 6,0 & 8,3 & 6,5 & 7,8 & 2,7 & 2,4 & 7,9 & 12,3 \\
\hline 1981 & 4,0 & 12,2 & 5,0 & 23,7 & $9,3 \quad 28,6$ & 13,0 & 30,4 & 8,2 & 10,7 & 7,4 & 8,2 & 4,7 & 5,3 & 2,6 & 2,3 & 7,1 & 11,5 \\
\hline 1982 & 6,5 & 20,3 & 8,6 & 29,1 & $9,6 \quad 26,6$ & 11,0 & 23,9 & 10,1 & 12,5 & 6,7 & 7,6 & 6,9 & 9,8 & 3,2 & 3,1 & 8,1 & 13,4 \\
\hline 1983 & 7,6 & 23,4 & 8,3 & 21,6 & $10,429,7$ & 13,3 & 24,3 & 11,0 & 13,8 & 9,0 & 11,0 & 2,2 & 2,6 & 4,3 & 3,6 & 8,8 & 13,6 \\
\hline 1984 & 5,3 & 17,8 & 7,1 & 21,9 & $6,9 \quad 21,7$ & 9,4 & 20,2 & 9,8 & 11,5 & 6,8 & 7,7 & 5,8 & 7,2 & 3,0 & 2,6 & 7,1 & 11,2 \\
\hline 1985 & 6,0 & 17,4 & 3,9 & 14,0 & $5,7 \quad 23,4$ & 9,2 & 20,0 & 6,5 & 9,3 & 6,6 & 7,3 & 4,2 & 5,2 & 1,7 & 2,1 & 5,8 & 9,6 \\
\hline 1986 & 2,5 & 10,3 & 4,2 & 16,1 & $9,4 \quad 33,9$ & 6,0 & 12,5 & 4,8 & 5,2 & 2,3 & 2,3 & 7,4 & 8,0 & 3,9 & 3,2 & 4,9 & 7,4 \\
\hline 1987 & 3,9 & 17,0 & (...) & 21,2 & (...) 22,6 & $(\ldots)$ & 13,3 & (...) & 6,6 & (...) & 4,8 & $(\ldots)$ & 3,6 & $(\ldots)$ & 1,4 & 4,8 & 7,5 \\
\hline 1988 & 3,8 & 16,0 & 2,7 & 9,1 & $5,6 \quad 23,5$ & 4,7 & 14,5 & (...) & 7,1 & $(\ldots)$ & 5,3 & (...) & 3,9 & $(\ldots)$ & 2,6 & 5,1 & 7,9 \\
\hline 1989 & 3,8 & 14,9 & 8,2 & 23,2 & $8,6 \quad 26,0$ & 9,7 & 13,5 & 5,9 & 5,1 & 5,4 & 4,6 & 3,7 & 4,3 & 4,1 & 3,6 & 6,2 & 8,1 \\
\hline 1990 & 4,7 & 29,2 & 3,4 & 20,5 & $4,0 \quad 20,5$ & 9,0 & 12,7 & 4,3 & 4,8 & 3,6 & 4,6 & 3,0 & 4,2 & 1,5 & 1,8 & 4,4 & 7,6 \\
\hline 1991 & 2,5 & 14,7 & 5,3 & 22,4 & $5,2 \quad 23,2$ & 9,8 & 10,3 & 6,7 & 4,9 & 4,1 & 4,6 & 3,7 & 3,9 & 2,6 & 2,5 & 5,2 & 6,5 \\
\hline 1992 & 3,9 & 14,5 & 2,4 & 10,0 & $7,4 \quad 25,3$ & 7,4 & 8,3 & 3,8 & 3,5 & 2,5 & 2,9 & 2,1 & 2,6 & 2,2 & 2,0 & 4,3 & 5,5 \\
\hline 1993 & 1,9 & 6,8 & 1,9 & 9,6 & $6,4 \quad 25,8$ & 9,4 & 8,8 & 5,2 & 4,2 & 3,9 & 4,4 & 5,6 & 6,3 & 1,7 & 1,7 & 4,8 & 5,9 \\
\hline 1994 & 4,6 & 21,7 & 3,8 & 17,2 & $5,6 \quad 21,4$ & 10,4 & 9,5 & 4,9 & 3,7 & 4,7 & 4,6 & 4,0 & 4,1 & 2,1 & 1,8 & 5,3 & 6,0 \\
\hline
\end{tabular}

para o ano de 1994. No ano de 1991, 88,2\% dos óbitos foram do sexo masculino e em 1994 esse valor foi de $86,4 \%$. O coeficiente especifico de mortalidade por afogamento no sexo masculino no município de Salvador diminuiu de 9,2 em 1991 para 7,6 em 1994 e no sexo feminino de 1,1 para 0,9.

Pode-se observar que para o sexo feminino, no ano de 1991, houve um maior risco de morte na faixa de dez a 14 anos seguida pela de 20 a 29 anos e 5 a 9 anos. No ano de 1994, assim como no sexo masculino a faixa etária de 15 a 19 anos atingiu um maior risco (Tabela 2).
Os maiores percentuais de mortes por afogamento ocorreram no periodo de outubro a fevereiro em ambos os anos. No ano de 1991, verificaram-se maiores percentuais nos meses de fevereiro (14,7\%), outubro (14,7\%) e dezembro (15,7\%); já no ano de 1994, as maiores freqüências corresponderam aos meses de janeiro $(17,3 \%)$, outubro $(14,4 \%)$ e dezembro $(12,5 \%)$.

Em relação ao grau de instrução das vitimas, verificaram-se maiores percentuais das que possuiam o $1^{\circ}$ grau, $72,5 \% \mathrm{em} 1991 \mathrm{e}$ $63,36 \% \mathrm{em} 1994$.

Tabela 2 - Número de óbitos e coeficiente de mortalidade/100.000 habitantes por afogamento por sexo e faixa etária, Salvador, 1991 e 1994.

\begin{tabular}{|c|c|c|c|c|c|c|c|c|c|c|c|c|}
\hline \multirow{3}{*}{$\begin{array}{l}\text { Faixa } \\
\text { Etária }\end{array}$} & \multicolumn{8}{|c|}{ Sexo } & \multicolumn{4}{|c|}{ Total } \\
\hline & \multicolumn{2}{|c|}{1991} & \multicolumn{2}{|c|}{1994} & \multicolumn{2}{|c|}{1991} & \multicolumn{2}{|c|}{1994} & \multicolumn{2}{|c|}{1991} & \multicolumn{2}{|c|}{1994} \\
\hline & $\mathrm{N}^{\circ}$ & $\mathbf{C M}^{(1)}$ & $\mathrm{N}^{\mathrm{o}}$ & CM & $\mathrm{N}^{\mathrm{o}}$ & $\mathrm{CM}$ & $\mathrm{N}^{\mathrm{o}}$ & CM & $\mathbf{N}^{\mathbf{o}}$ & CM & $\mathbf{N}^{\mathbf{o}}$ & $\mathrm{CM}$ \\
\hline $0-4$ & 3 & 2,9 & 5 & 5,2 & 1 & 1,0 & 1 & 0,9 & 4 & 2,0 & 6 & 3,0 \\
\hline $5-9$ & 11 & 8,9 & 8 & 6,0 & 2 & 1,7 & 2 & 1,5 & 13 & 5,3 & 10 & 3,8 \\
\hline $10-14$ & 12 & 9,7 & 10 & 7,5 & 3 & 2,3 & 2 & 1,5 & 15 & 6,0 & 13 & 4,8 \\
\hline $15-19$ & 20 & 19,4 & 21 & 19,0 & 1 & 0,8 & 3 & 2,3 & 21 & 9,4 & 24 & 10,0 \\
\hline $20-29$ & 18 & 9,3 & 16 & 7,7 & 4 & 1,8 & 1 & 0,4 & 22 & 5,3 & 17 & 3,8 \\
\hline $30-39$ & 14 & 9,5 & 10 & 6,3 & - & - & 2 & 1,1 & 14 & 4,4 & 13 & 3,8 \\
\hline $40-49$ & 6 & 6,8 & 6 & 6,3 & - & - & - & - & 6 & 3,2 & 6 & 3,0 \\
\hline $50 E+$ & 6 & 6,5 & 3 & 3,0 & 1 & 0,7 & - & - & 7 & 3,1 & 3 & 1,2 \\
\hline TOTAL & 90 & 9,2 & 79 & 7,6 & 12 & 1,1 & 11 & 0,9 & 102 & 4,9 & 92 & 4,3 \\
\hline
\end{tabular}

(1) CM - coeficiente de mortalidade. 
Os DS Itapuã, Boca do Rio e Cajazeiras foram as áreas com maiores taxas de mortalidade por afogamento em Salvador no ano de 1991: 10,6; 9,9 e 9,0 óbitos por 100.000 babitantes, respectivamente. No ano de 1994, o quadro mudou, ou seja, São Caetano/Valéria $(6,7)$, Pau da Lima $(6,5)$ e Cabula/Beiru $(5,7)$ tiveram os maiores coeficientes. Pode-se observar que houve um aumento no risco de morte por afogamento nos residentes dos DS São Caetano/ Valéria (6,2 para 6,7), Cabula/Beiru (3,5 para 5,7), Pau da Lima (4,9 para 6,5) e Subúrbio Ferroviário (3,0 para 3,4). (Figuras 1 e 2).
$\mathrm{Na}$ distribuição dos afogamentos segundo local de ocorrência, chama atenção a grande parcela que representa os óbitos onde esses locais são ignorados, para o ano de 1991 (9,8\%), assim como para o ano de 1994 (11,4\%). No ano de 1994, é grande a parcela de óbitos de residentes de Salvador ocorridos em outros municípios (21,9\%). O distrito sanitário onde mais se morre por afogamento é o de Itapuã: 28,4\%, em 1991, e 27,1\%, em 1994. Os distritos Barra/Rio Vermelho (9,8\%/1991; 10,4\%/1994) e Subúrbio Ferroviário (7,8\%/ 1991; 8,3\%/1994) possuem percentuais altos.

Figura 1 - Coeficiente de mortalidade por afogamento segundo distrito sanitário da residência da vítima, Salvador, 1991

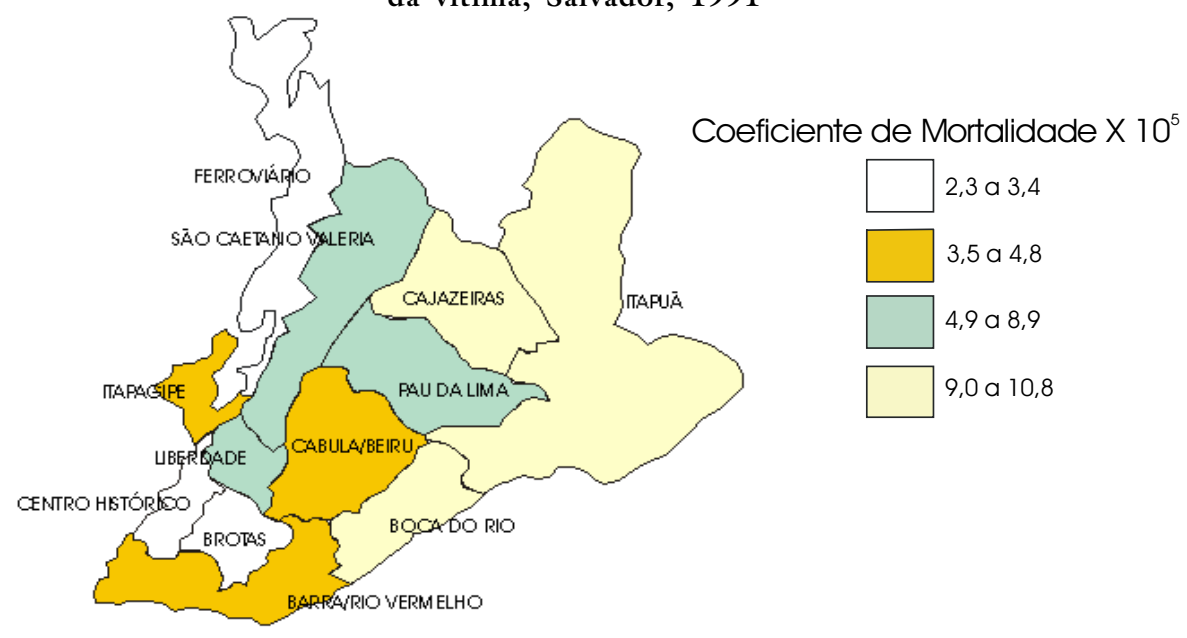

Figura 2 - Coeficiente de mortalidade por afogamento segundo distrito sanitário da residência da vítima, Salvador, 1994

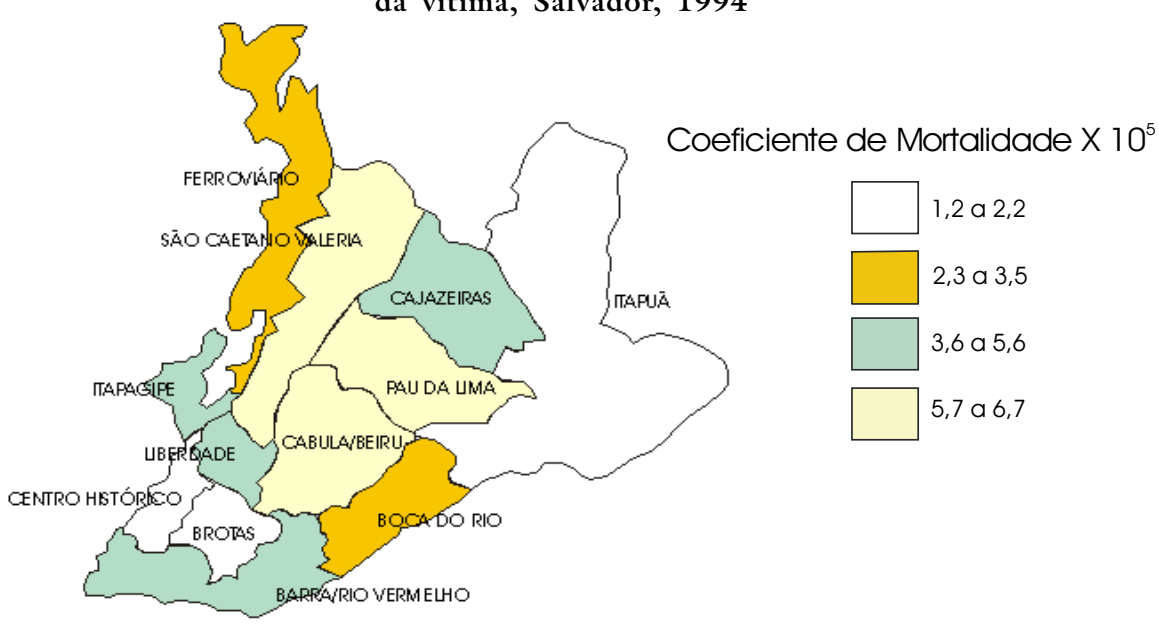


Nas Figuras 3 e 4 observa-se que os afogamentos dão uma maior contribuição para a mortalidade por causas externas nos DS do litoral de Salvador, principalmente em Itapuã (28,4 e 28,6\%), Boca do Rio (21,4 e 14,3\%) e Barra/Rio Vermelho (8,1 e 8,0\%).

Em relação à ocupação das vitimas, os estudantes tiveram os maiores percentuais, $32,4 \%$ e 40,6\%, em 1991 e 1994, respectivamente. Nenhuma outra ocupação teve destaque.

\section{Discussão}

Apesar do declínio do risco de morte por afogamento em Salvador nos anos estudados, esses dados mostram-se superiores às taxas observadas em outros locais, com exceção do Alaska. Nos Estados Unidos, por exemplo, a taxa de mortalidade por afogamento variou de 3,9 em 1970 a 1,7/100.000 habitantes em 1995. ${ }^{5}$ Para esses mesmos anos, no Arizona, essas taxas declinaram de 5,7 para 2,6.5 Um estudo realizado na Austrália para o ano de 1994

Figura 3 - Mortalidade proporcional por afogamento em relação a causas externas segundo local de ocorrência, Salvador, 1991

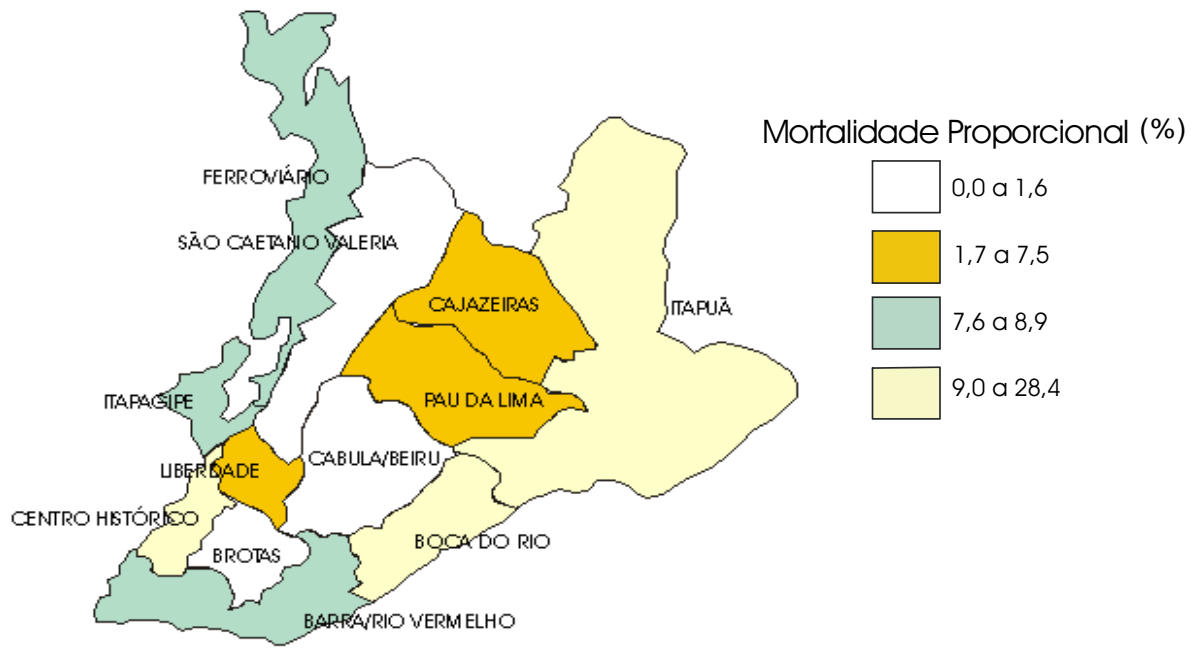

Figura 4 - Mortalidade proporcional por afogamento em relação a causas externas segundo local de ocorrência, Salvador, 1994

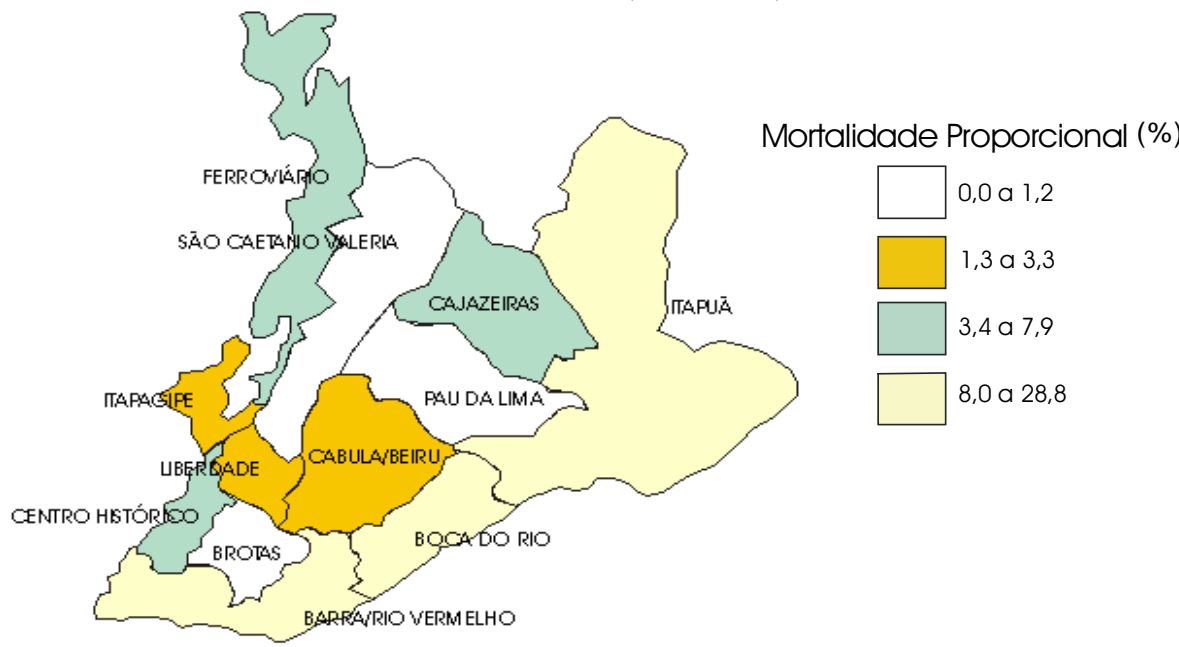


revelou uma taxa de 1,4/100.000 babitantes em toda essa região. ${ }^{4}$ Para o município do Rio de Janeiro, em 1996, a taxa total de mortalidade foi de 2,2. Em Salvador, a taxa de mortalidade foi de 4,9 para 1991 e de 4,3 em 1994, variando de 0,4 a 9,8, de acordo com as áreas programáticas. ${ }^{17}$

Por outro lado, no Estado do Alaska, afogamento é a maior preocupação de saúde pública. Um estudo realizado neste local no periodo de 1988 a 1992 revelou uma taxa de incidência anual de 20 afogamentos por 100.000 habitantes. ${ }^{6}$ Enquanto no presente estudo foram verificadas maiores taxas para a faixa etária de 15 a 19 anos, no Alaska o grupo de idade de 20 a 39 anos teve as maiores freqüências e taxas de afogamento. Neste Estado, as vitimas estão mais relacionadas a pescadores e jovens nativos enquanto no municipio de Salvador não foi detectado nenhum óbito que tivesse como ocupação pescador ou similar.

Como mencionado nos resultados, houve diferença entre o número de óbitos obtidos após revisão do banco de dados do Instituto de Saúde Coletiva (ISC) em relação aos dados oficiais do Ministério da Saúde. Uma explicação possivel para essa diferença é que os casos de óbito de residentes de outros municípios ocorridos em Salvador foram retirados do banco de dados final do ISC.

Foi observado um declínio da contribuição dos óbitos por afogamento na mortalidade por causas externas a partir dos 20 anos de idade. Isso pode estar relacionado à maior importância dos homicídios e dos acidentes de trânsito, ${ }^{2,3}$ em idades mais avançadas, neste grupo de causas.

Confirmando o que já foi observado em vários estudos $4,6,7,8,9,10,18,19$ bouve uma predomináncia do sexo masculino entre as vitimas de afogamento em todas as idades e a ocupação de destaque foi a de estudantes, com números bem superiores aos das outras profissões, refletindo, provavelmente, a alta exposição desse grupo. Nos dois anos em estudo a maioria das vitimas só tinba o primeiro grau de escolaridade o que aponta para a importância da educação nesse tipo de morte. Houve uma concentração dos óbitos nos meses de verão e nos DS pertencentes ao litoral de Salvador.

Mello Jorge ${ }^{18,19}$ levanta a bipótese de que os homens se expõem mais a esse tipo de risco, em geval por recreação. A preponderância do sexo masculino e da faixa etária com maior risco pode estar relacionada também à ingestão de álcool apontada em alguns estudos ${ }^{12,18}$ chegando a relatos de que $61 \%$ das vitimas haviam feito uso desta substância. ${ }^{18}$

A elevada freqüência desse tipo de acidente durante os meses de verão decorre da maior utilização das praias pela populalação nesse periodo. Da mesma forma, no Arizona e no Alaska 6 a maior parte dos óbitos ocorreu nessa estação.

No que se refere à distribuição espacial, os resultados das taxas de mortalidade por afogamento relacionados ao local de residência da vitima expressam a heterogeneidade do municipio de Salvador, revelando uma desigualdade no risco de morrer afogado entre os moradores dos diferentes locais da cidade.

Tendo-se conbecimento da dimensão do problema e das principais características da mortalidade por afogamento no município de Salvador, pode-se formular estratégias de prevenção e tratamento das vitimas, com ênfase nos grupos de maior risco.

Trabalhos realizados na área pediátrica ${ }^{10,17}$ aconselham a estimular crianças a aprenderem a nadar. Saber nadar é a regra básica na prevenção de acidentes na água, e uma criança de três anos de idade normalmente já tem condiçôes de aprender. Não menos importante é a supervisão do adulto e a observância às normas de segurança.

Em relação à assistência hospitalar, existem evidências segundo as quais o atendimento correto e o mais precoce possivel, prestado desde o local do acidente até a chegada ao hospital, pode diminuir as sequelas dos acidentados. ${ }^{11,12}$ No Estado do Alaska foram 
desenvolvidos programas para venda de bóias salva-vidas a baixo custo. Há relatos de que 16 pessoas da região no periodo de um ano atestaram que o uso das bóias foi o fator responsável pelo salvamento de suas vidas. ${ }^{18}$

$O$ elevado risco de morrer por afogamento, particularmente entre adolescentes do sexo masculino e estudantes, indica a necessidade da adoção de medidas voltadas para o controle desse problema que priorizem esses grupos. Dai a pertinência de desenvolver campanhas de esclarecimento a essa população sobre os perigos das praias localizadas nos DS de maiores taxas de mortalidade, implantar programas educativos nas escolas e aperfeiçoar o serviço de salva-vidas nos meses de verão e nos locais de maior risco. A profilaxia realizada por estes profissionais nas praias, com sua intervenção precoce, pode ser mais eficiente que a otimização do atendimento tardio hospitalar. ${ }^{12}$

Por fim, merece destaque o papel que os DS podem desempenhar através da gerência e das equipes de saúde. Esse é um exemplo de agravo à saúde evitável, porém cujo controle requer a adoção de politicas intersetoriais envolvendo, além do setor saúde, outros órgãos governamentais como Corpo de Bombeiros e Universidades, além de associações representativas da população.

\section{Bibliografia}

1. Organização Mundial da Saúde (OMS). Classificação Internacional de Doenças. Revisão 1975. Centro da OMS para classificação de doenças em português. Ministério da Saúde/Universidade de São Paulo/Organização Pan-Americana de Saúde. São Paulo, 1985. 800p.

2. Centro Brasileiro para a Infáncia e Adolescência - CBIA e Fundo das Nações Unidas para a Infancia - UNICEF. Babia: suas Crianças e Adolescentes. O que está acontecendo?, p.99-121, 1991.

3. BAHIA, Secretaria da Justiça e Direitos Humanos, Sistema de Vigiláncia da Violência Contra a Criança e o Adolescente e Fundo das Nações Unidas para a Infäncia
- UNICEF. Mortes Violentas de Crianças e Adolescentes em Salvador, p. 22-37, 1993.

4. National Injury Surveillance Unit-Australian Institute of Health \& Welfare. Drowning deaths. Bulletin 13. Autralia 1994 (on-line), disponivel em < URL: http://www.nisu. flinders.edu.au/pubs/bulletin 13/bull135.html (28/05/98).

5. Public Health Services - Office of Health Planning, Evaluation and Statistics Arizona Center for Health Statistics. Unintentional Drowning Deaths, Arizona, 1970-1995: Table 8.(on-line), disponivel em < URL: http://www.hs.state.az.us/plan/drowning/ tables/drt8.htm and http:// www.hs.state.az. us/plan/drowning/tables/drt17btm (28/05/ 98).

6. Lincoln JM, Perkins R, Melton F, Conway GA. Drowning in Alaskan Waters. Public Health Reports 111(6):531-535, 1996.

7. Szpilman D, Amoedo AR. Atualização da classificação de afogamento. Jornal Brasileiro de Medicina, 66(4):21-37, 1994.

8. Jiménez NR. Asfixia por Inmersión. Pediatria. 32(1):56-60, 1989.

9. Jiménez NR, Coronado L. Asfixia por Inmersión en Niños 1983-1988. Revista del Hospital del Niño 9(1):33-38, 1990.

10. Rivera JF, Gotay F, Sifontes JE, Mayol PM. Near Drowning in Puerto Rican Children. Asociación Médica de Puerto RicoBoletin 79(3):95-98, 1987.

11.Schiling ALD, Garibotti JE, Santos de Moraes MR, Salerno MR. Prevenção de acidentes na infância. ACTA MED 15:641$656,1994$.

12. Szwarcwald CL, Castilho EA. Mortalidade por Causas Externas no Estado do Rio de Janeiro no periodo de 1976 - 1980, Cadernos de Saúde Pública, 2(1):1941, 1986.

13.BRASIL, Estatisticas de Mortalidade do Ministério da Saúde, 1980 - 1994. 
14.Paim JS, Vieira da Silva LM, Nascimento Costa MC, Lessa I, Costa Cardoso AJ, Pinto LLS, Xavier Calil ME, Barreto da Silva MRB, Mascarenhas JCS, Pereira SV. Análise da situação de saúde do município de Salvador segundo condiçôes de vida. Relatório Técnico do Projeto Integrado de Pesquisa.

15. Paim JS, Costa MCN. Mortes Violentas em Crianças e Adolescentes de Salvador. Revista Bahia, Análise e Dados 6(1):5967, 1996.

16.Paim JS, Costa MCN. Decréscimo e Desigualdade da Mortalidade Infantil: Salvador, 1980 - 1988. Boletin de la Oficina Sanitaria Panamericana
114(5):415-428, 1993.

17.Souza ER, Assis SG. Mortalidade por Violência em Crianças e Adolescentes do Municipio do Rio de Janeiro. Jornal Brasileiro de Psiquiatria 42(2):85-94, 1996.

18. Mello Jorge, $\mathrm{MH}$ e Bernardes Marques, $M$. Mortes Violentas em Menores de 15 Anos no Brasil. Boletim da Oficina Sanitária Panamericana 100(6):590-606, 1986.

19.Mello Jorge MH. Mortalidade por Causas Violentas no Municipio de São Paulo. Mortes Acidentais. Revista de Saúde Pública 14:475-508, 1980. 